\title{
Cerebral infarction in young people. A study of 148 patients with early cerebral angiography
}

Fabrice Lisovoski, Pascal Rousseaux

\begin{abstract}
The aetiology of strokes was studied in a hospital based series of patients aged up to 40 years with precise clinical and radiological criteria. One hundred and forty five patients $(75$ males and 73 females) aged five to 40 years with cerebral ischaemia were evaluated. Aetiology was heterogeneous and could be classified into seven groups. Cerebral arteriograms were performed in all cases and indicated the aetiological diagnosis in most patients. Embolism was the most frequent recognised abnormality $(38 \cdot 4 \%)$. There were no complications of arteriography. Arterial dissections discovered by arteriography were the cause of the stroke in $10.1 \%$ of the patients. Atherosclerosis was diagnosed in 32 cases and was the commonest cause $(21 \cdot 6 \%)$. In one fifth of cases no cause was found. Contraceptive drugs were considered as potential cause of ischaemic stroke in $11.5 \%$, cardiac diseases in $12.8 \%$ and haematological disorders in $8.1 \%$. Other potential causes included migraine, inflammatory diseases, pregnancy and lacunas. Follow up in 126 cases showed that many patients had good functional recovery.
\end{abstract}

Ischaemic stroke occurs predominantly in elderly adults. Atherosclerosis is known to be the common aetiology. About three per cent of cerebral infarctions occur in young individuals. ${ }^{1}$ The aetiology of ischaemic stroke in older patients differs from that of younger subjects due to differences in genesis of cerebrovascular diseases in the two populations. For instance, cerebral infarction in young people may be associated with lupus anticoagulants or with the use of oral contraceptive drugs. A number of reports regarding aetiological analysis of cerebral infarction in young adults are available. Few studies, however, have included more than 100 patients. ${ }^{2-4}$ Moreover, cerebral arteriograms have not always been performed. We report a study of 148 patients under the age of 40 with cerebral infarction including early cerebral arteriograms.

\section{Patients and methods}

One hundred and forty eight patients (75 males and 73 females) aged five to 40 years (median age 32) who were admitted to hospi- tal for non-haemorrhagic arterial cerebral infarction during 1 January 1976 to June 30 1988 were reviewed retrospectively. As this is both a neurological and neurosurgical department, 698 patients were admitted for ischaemic stroke during this period. Brain ischaemia diagnosis was verified retrospectively. Amongst the 148 patients under 40 years of age, seven $(4.7 \%)$ had transient ischaemic attacks. Forty six per cent of the patients had two dimensional echocardiograms, and $83.1 \%$ had CT scans. Cerebral arteriograms were performed in all cases. One hundred and forty six out of the 148 arteriograms $(98.6 \%)$ were performed within eight days after the onset of the stroke, 114 $(77 \%)$ were made within three days. There were no complications due to angiographic investigations.

Risk factors for cerebrovascular disease were noted for each individual aetiology. Hypertension was defined as blood pressure greater than $160 / 95 \mathrm{~mm} \mathrm{Hg}$ recorded on three occasions during admission to hospital or in patients previously treated for hypertension. Diabetes mellitus was generally diagnosed before the onset of cerebral infarction. Hyperlipidaemia was defined as an excess in serum cholesterol (more than $10 \mathrm{mmol} / \mathrm{l}$ ) with or without hypertriglyceridaemia. The index for being overweight was considered to be $15 \%$ above the ideal weight. Smoking was noted as a risk factor beyond consumption of 15 cigarettes daily.

A probable cause of ischaemic stroke was diagnosed on the basis of clinical, biological and radiological data. Atherosclerosis was considered contributory if typical lesions were found on arteriography. This cause was also presumed if the patient had three or more risk factors leading to atherosclerosis in cases where no arteriographic lesions or identifiable causes were detected. Besides the usual haematological tests, antithrombin III, coagulating factors and platelet activity were measured in the patients having haematological abnormalities. Blood viscosity and fibrinolysis were studied in six patients. $C$ and also $S$ protein were measured in five cases. Circulating anticoagulants were looked for in three cases. Tissue plasminogen activator was measured in two patients. The use of oral contraceptives was considered as a cause and not just a risk factor for cerebral infarction in the absence of any known aetiology leading to brain ischaemia. We attributed cerebral infarction to migraine only in cases of classic migraine. Despite diagnostic investigations, 
Table 1 Distribution of age and sex ratio

\begin{tabular}{llll}
\hline Age & Females & Males & Sex ratio $F / M$ \\
\hline$<=20$ & 7 & 6 & $1 \cdot 17$ \\
$21-25$ & 10 & 3 & $3 \cdot 33$ \\
$26-30$ & 16 & 6 & $2 \cdot 67$ \\
$31-35$ & 16 & 24 & $0 \cdot 67$ \\
$36-40$ & 24 & 36 & $0 \cdot 67$ \\
\hline
\end{tabular}

many cases of cerebral infarction were found to be without a clear aetiology. We have termed these cases "undetermined causes".

Follow up information regarding the course of functional recovery was obtained either by consultation or correspondence in 126 cases $(85 \cdot 1 \%)$ with a 27 month mean follow up. The outcome was defined as favourable when functional recovery was complete or when the remaining disability was moderate.

\section{Results}

Amongst the 148 patients in this study, 11 had multiple ischaemic strokes. The final number of cerebral infarctions was 161 (80 in males, 81 in females). Sex and age of the 148 patients are shown in table 1 . Over the age of 30 , males were predominant. In the age group 21-30, the sex ratio $F / M$ was significantly reversed (Chi square $=12.51,0.01<\mathrm{p}<0.02)$, probably due to the use of oral contraceptive drugs by females.

Cerebral arteriograms were abnormal in 98 cases $(66 \cdot 2 \%)$. The detailed results are reported in table 2. The classification of arterial lesions was made on the basis of angiographic appearances. One patient had two dissections. The most frequent recognised abnormality was embolism $(38.4 \%)$ followed by thrombosis $(28.3 \%)$. Atherosclerosis and arterial dissections were diagnosed respectively in $17 \cdot 2 \%$ and $16 \cdot 1 \%$ of the remaining cases. Seventy nine point four per cent of all vascular lesions were in the carotid territory and $20.6 \%$ in the basilar territory. In thrombosis and arterial dissections groups, $75 \%$ of the vascular lesions were recognised in the carotid territory and $25 \%$ in the basilar territory. On the other hand, $94.7 \%$ of the lesions which were diagnosed as embolism were found in the carotid territory and $5.3 \%$ in the basilar territory.

Atherosclerosis was diagnosed in 32 cases $(21 \cdot 6 \%)$. Seventeen patients had typical lesions of atherosclerosis, as shown by arteriography. The other patients in this group had at least three common risk factors for cerebrovascular disease. These factors were: smoking (19), high blood pressure (10),

Table 2 Detailed results of arteriographic lesions

\begin{tabular}{|c|c|c|c|c|}
\hline & Embolism & Thrombosis & Dissection & Atherosclerosis \\
\hline $\begin{array}{l}\text { Territory } \\
\text { Number }\end{array}$ & $\begin{array}{l}\text { Carotid/Basilar } \\
36 \quad 2\end{array}$ & $\begin{array}{l}\text { Carotid/Basilar } \\
21\end{array}$ & $\begin{array}{l}\text { Carotid/Basilar } \\
12 \\
4\end{array}$ & $\begin{array}{l}\text { Diffuse } \\
17\end{array}$ \\
\hline Total & 38 & 28 & 16 & 17 \\
\hline
\end{tabular}

overweight (10), coronary disease (eight), hyperlipidaemia (eight), diab̄etes mellitus (five), leg claudication (two). Sex ratio and median age are listed in table 3.

Heart disease was the likely cause in a group of 19 patients $(12 \cdot 8 \%)$. The risk factors for cerebrovascular disease were: high blood pressure (three cases), smoking (three), overweight (one), migraine (one), oral contraceptives (one). The following cardiac disorders were recognised: valvular heart disease (found in six), cardiomyopathy (three), prosthetic valve (three), idiopathic arrhythmia (three), atrial septal aneurysm (two), infectious endocarditis (one), Wolf-Parkinson-White disease (one). Isolated mitral valve prolapse was not accepted as a cause of cerebral infarction.

Twelve patients $(8 \cdot 1 \%)$ had haematologically related disease. Recognised blood disorders were as follows: thrombocytosis (three), blood hyperviscosity (two), platelet hyperaggregation (two), C protein deficiency (one), tissue palsminogen activator decrease (one), factor VIII increase (one), thrombopenia due to heparin treatment (one), circulating anticoagulant (one). Associated risk factors for this aetiology were: smoking (four), migraine (three), contraceptive drugs (two).

Ischaemic stroke was attributed to oral contraceptives in 17 females $(11.5 \%)$ whose associated risk factors were: migraine (seven), smoking (three), overweight (two), hyperlipidaemia (one).

Arterial dissection was the cause of the stroke as shown by arteriography in 15 cases $(10 \cdot 1 \%)$. Because one patient had a double lesion, 16 arterial dissections were diagnosed. Carotid arteries were damaged in 12 cases and the vertebral arteries in four cases. Considered risk factors: oral contraceptives (five), migraine (four), high blood pressure (three), smoking (three), hyperlipidaemia (one), overweight (one).

Thirty patients $(20.3 \%)$ could not be classified in any cited aetiology. Two had a double stroke. These patients presented with the following risk factors: migraine (seven), overweight (three), diabetes mellitus (two), smoking (two), hyperlipidaemia (two), coronary disease (two), transient ischaemic attack (two).

Eighty four and a half per cent of the patients belong to these six groups. The remaining $15.5 \%$ are listed as "other causes" including inflammatory diseases (six patients), lacunar disease (six), pregnancy (four), migraine (three), infection (two), postoperative (two). Migraine was the presumed cause of stroke in three females, in whom the usual aura evolved into a permanent defect. The associated risk factors were: overweight (six), high blood pressure (five), smoking (four), diabetes mellitus (three), hyperlipidaemia (two), oral contraceptives (one).

Percentage of favourable outcomes in each aetiology is specified in table 3 . Eighty three of the 126 patients $(65.9 \%)$ who were followed up showed favourable outcome. Seven $(5 \cdot 5 \%)$ patients died. 
Table 3 Distribution of sex ratio, median age and percentage of favourable outcomes in each aetiology

\begin{tabular}{lllll}
\hline Aetiology & $N$ & Sex ratio F/M & Median age & $\begin{array}{l}\text { Favourable } \\
\text { outcome (\%) }\end{array}$ \\
\hline Atherosclerosis & 32 & $0 \cdot 28$ & $35 \cdot 9$ & 60 \\
Cardiac causes & 19 & $0 \cdot 73$ & $29 \cdot 4$ & $73 \cdot 3$ \\
Haematological disorders & 12 & 2 & 29 & $54 \cdot 5$ \\
Oral contraceptives & 17 & 2 & $31 \cdot 2$ & $64 \cdot 3$ \\
Dissections & 15 & 2 & $34 \cdot 7$ & $46 \cdot 1$ \\
Undetermined causes & 30 & $0 \cdot 36$ & $29 \cdot 8$ & 76 \\
Other causes & 23 & $0 \cdot 67$ & 31.9 & $88 \cdot 9$ \\
\hline
\end{tabular}

\section{Discussion}

Ischaemic stroke in young people is not a rare pathology. In patients over 45 years old, atherosclerosis is known as the predominant cause of ischaemic stroke. The incidence of cerebral infarctions due to atherosclerosis doubles every ten years. On the contrary, the potential causes of ischaemic strokes in young people are numerous. Several new causes have been recently identified, such as paradoxical embolism, ${ }^{56}$ Eales' disease, ${ }^{78}$ infection, ${ }^{9}$ lupus anticoagulant ${ }^{10}$ or mitochondrial encephalomyopathy. ${ }^{11-13}$ The lack of consensus about aetiological criteria has led to varying percentages of causal factors in reported series. ${ }^{23}$ Two accredited risk factors for atherosclerosis, in the absence of any other identifiable cause, have been defined by some authors ${ }^{23}$ as sufficient basis for a presumed cause of atherosclerosis. In such studies, undetermined causes were reduced in favour of atherosclerosis. We report here a classification of ischaemic stroke aetiology where three risk factors were necessary for the aetiological diagnosis of atherosclerosis which diminished its prevalence compared with studies where only two risk factors were necessary. This study aimed at establishing precise percentages of the aetiological origin of ischaemic accidents in young people using precise clinical and radiological criteria. Our classification included seven groups (table 4): atherosclerosis, cardiac causes, haematological causes, oral contraceptives, arterial dissections, other and undetermined causes.

Atherosclerosis represented the main cause $(21.6 \%)$ of cerebral ischaemia in young people. In this study, $77 \%$ of cerebral arteriograms were made within three days. This fact substantially reduced the risk that originally occluded vessels by an embolism had undergone recanalisation during this delay. Thus it seemed appropriate that the patients having normal cerebral angiography and at least three risk factors for atherosclerosis were considered to have stroke due to atherosclerosis.

A cardiac cause was diagnosed in $12.8 \%$ of the patients. Electrocardiogram (ECG), two-

Table 4 Distribution of aetiologies of cerebral infarction among 148 patients under the age of 40

\begin{tabular}{lccc}
\hline Atherosclerosis & Undetermined causes & $\begin{array}{l}\text { Other causes } \\
15 \cdot 5 \%\end{array}$ & $\begin{array}{c}\text { Cardiac causes } \\
21.6 \%\end{array}$ \\
$20.3 \%$ & & Haematological disorders \\
Oral contraceptives & Arterial dissections & & $8 \cdot 1 \%$ \\
$11.5 \%$ & $10 \cdot 1 \%$ & & \\
\hline
\end{tabular}

dimensional echocardiography and ECG recording by Holter method during 24 hours are not considered sufficient for a complete cardiac evaluation, which requires contrast echocardiographic investigations. Unfortunately, as our study was retrospective, none of the patients had contrast echocardiographic records. It is known that contrast echocardiography allows detection of right-to-left shunts. ${ }^{14}$ But this cardiac anomaly seems to be a common event in the healthy population..$^{15}$ Patent foramen ovale has been found more frequently among patients with cerebral infarction compared with a healthy group. ${ }^{56}$ It appears necessary, however, to determine the origin of the embolism to confirm the diagnosis of paradoxical embolism. Thus patent foramen ovale was considered as the real cardiac aetiology of ischaemic stroke in only $4 \%$ of the cases reported by Adams et $\mathrm{al}^{2}$ and in less than $1 \%$ in the study conducted by Gautier et al. ${ }^{3}$ The fact that this study was conducted without contrast echocardiographic investigations does not significantly modify our conclusions about the classification.

The group of haematological disorders consisted of $8 \cdot 1 \%$ of the patients. Several of these abnormalities are well recognised as causes of cerebral ischaemia. It was shown that platelet hypercoagulability could underlie strokes in young adults. ${ }^{19}$ Recent studies on platelet activity or coagulation allow the recognition of newly described haematological causes of ischaemic stroke.

Cerebral infarction is a potential complication amongst females taking contraceptive drugs. ${ }^{20-22}$ Recent immunological studies showed anti-ethynyloestradiol antibodies in the blood of most females with brain infarction after using contraceptive drugs. ${ }^{23-26}$ Arterial lesions were found by radiological and histological investigations, ${ }^{27} 28$ indicating that the use of oral contraceptives is in itself a cause and not a simple risk factor for cerebral ischaemia. No relation was found between the duration of the use of the contraceptives and cerebrovascular complications. Nevertheless, oral contraceptive-induced cerebral ischaemia should remain a diagnosis of exclusion. In our population migraine was found among $41.2 \%$ of the females whose cerebral ischaemia was attributed to oral contraceptives. Since smoking was found in only $17 \cdot 6 \%$ of the cases, these results suggest that oral contraceptive drugs when associated with migraine may be responsible for brain ischaemia than oral contraceptives associated with smoking.

Arterial dissection was found in $10.1 \%$ of the patients. None had recent neck injury. Thus we confirm the results of Adams $^{2}(6 \cdot 2 \%)$, which are lower than those of Gautier ${ }^{3}(20 \cdot 5 \%)$. The discrepancy could be due to a recruiting bias; that is, the existence of patients with dissection due to direct trauma which were ruled out in this study but not in that of Gautier et al. However, an indirect arterial traumatism is always possible, making it difficult to identify which dissections are truly spontaneous and which are not.

Migraine was the cause of cerebral infarction 
in three patients $(2 \%)$ although migraine was found in 25 cases $(16.9 \%)$. Ischaemic stroke is known to be a potential complication of migraine ${ }^{1617}$ but its relative contribution has yet to be precisely defined. Only classic migraine should be considered; the occurrence of a permanent deficit reproducing the usual aura is a conclusive argument that migraine is the real cause of ischaemic stroke. ${ }^{18}$

Of the cerebral arteriograms, $66 \cdot 2 \%$ were abnormal and allowed characterisations of arterial lesions that indicated the aetiological origin. The importance of arteriography in the evaluation of young patients with ischaemic stroke should be emphasised. It has to be performed early after the stroke because of the short duration of some radiological lesions such as embolism.

The highest percentage of favourable outcomes was found in the group of patients classified as "other causes" and "undetermined causes". The lowest percentage was found in the group presenting with arterial dissections. The patients with haematological disorders had severe neurological prognoses, as well as haematological complications. The results of this study on the prognosis must be viewed cautiously because this is not strictly a study but it confirms the relatively favourable outcome of brain infarctions among young people under the age of $40 .^{29}$

In conclusion, this study emphasises certain features concerning young people suffering from cerebral infarction: 1) The causes are more varied compared with an older population. Although the patients could be classified into seven major groups, the precise cause often remains uncertain. The undetermined causes are likely to decrease in the near future with the development of new diagnostic procedures such as new echocardiographic techniques or enhanced understanding of clotting mechanisms; 2) Among the patients in the 21-30 age group, females are predominant. The use of contraceptive drugs can explain this observation; 3) Cerebral arteriogram is an essential investigation for precisely evaluating cerebral ischaemia in young people. When performed early, it allows recognition of many arterial lesions that indicate the aetiological diagnosis; 4) The prognosis for functional recovery is favourable in most cases, since $65.9 \%$ of the patients showed functional recovery after a 27 month mean follow up.

1 Hart RG, Sherman DG, Miller VT, et al. Diagnosis and management of ischemic stroke: II. Selected controversies. Curr Probl Cardiol 1983;7:43-53.

2 Adams HP, Butler MJ, Biller J, Toffol GJ. Nonhemorrhagic cerebral infarction in young adults. Arch Neurol 1986;43:793-6.

3 Gautier JC, Pradat-Diehl P, Loron P, et al. Accidents vasculaires cérébraux des sujets jeunes. Une étude de 133 patients de 9 à 45 ans. Rev Neurol 1989;145:437-42.

4 Hart RG, Miller VT. Cerebral infarction in young adults: a practical approach. Stroke 1983;14:110-4.

5 Lechat P, Mas JL, Lascault G, et al. Patent foramen ovale and cerebral embolism (abstract). Circulation 1985: 72(suppl III): 134 .

6 Lechat P, Mas JL, Lascault G, et al. Prevalence of patent foramen ovale in patients with stroke. $N$ Engl J Med 1988;318:1148-52.

7 Gordon MF, Coyle PK, Golub B. Eales' disease presenting as stroke in the young adult. Ann Neurol 1988;24:264-6.

8 Masson C, Denis P, Prier S, Martin N, Cambier J. Maladie de Eales avec troubles neurologiques. Rev Neurol 1988;24:264-6.

9 Syrianen J, Valtonen VV, Iiavanainen $M$, et al. Preceding infection as an important risk factor for ischemic brain infarction in young and middle aged patients. $B M J$ 1988;296:1156-60.

10 Hart RG, Miller VT, Coull BM, Bril V. Cerebral infarction associated with lupus anticoagulants. Preliminary report. Stroke 1984;15:114-8.

11 Pavlakis SG, Phillips PC, Dimauro S, De Vivo DC, Rowland LP. Mitochondrial myopathy, encephalopathy, lactic acidosis and stroke-like episodes: a distinctive lactic acidosis and stroke-like episodes: a
clinical syndrome. Ann Neurol 1984;16:481-8.

12 Pavlakis SG, Phillips PC, Dimauro S, De Vivo DC, Rowland LP. Mitochondrial myopathy, encephalopathy, lactic acidosis and stroke-like episodes. Ann Neurol 1985;18:626.

13 Shapira Y, Harel S, Russell A. Mitochondrial encephalomyopathies. A group of neuromuscular disorders with defects in oxydative metabolism. Israel $J$ Med Sci 1977;13:161-4.

14 Lynch JJ, Schuchard GH, Gross CM, et al. Prevalence of right-to-left atrial shunting in a healthy population: detection by Valsalva maneuver contrast echocardiography. Am J Cardiol 1984;53:1478-80.

15 Hagen PT, Scholtz DG, Edwards DW. Incidence and size of patent foramen ovale during the first decades of life: an patent foramen ovale during the first decades of life: an
autopsy study of 965 normal hearts. Mayo Clinic Proc autopsy study

16 Hilton-Jones D, Warlow CP. The causes of stroke in the young. J Neurol 1985;232:137-43.

17 Hindfelt B, Nilsson O. Brain infarction in young adults. Acta Neurol Scand 1977;55:145-57.

18 Bousser MG, Baron JC, Chiras J. Ischemic strokes and migraine. Neuroradiology 1985;27:583-7.

19 Kalendovsky Z, Austin J, Steel P. Increased platelet aggregability in young patients with stroke. Arch Neurol 1975;32:13-20.

20 Collaborative group for the study of stroke in young women. Oral contraceptives and increased risk of cerebral ischemia or thrombosis. N Engl J Med 1973;228:871-8.

21 Lidegaard O. Cerebrovascular deaths before and after the appearance of oral contraceptives. Acta Neurol Scand appearance of oral

22 Lidegaard O, Soe M, Andersen MVN. Cerebral thromboembolism among young women and men in Denmark, 1977-1982. Stroke 1986;17:670-5.

23 Bakouche P, Vedrenne V, Beaumont V, Chaouat D, Reigner A. Thrombo-angéite à cellules géantes au cours d'une contraception orale-Etude anatomo-clinique et immunologique. Rev Neurol 1980;136:509-19.

24 Beaumont JL, Lemort N. Oral contraceptives, pulmonary artery thrombosis and anti ethynilestradiol Ig G. Clin Exp Immunol 1976;24:455-63.

25 Beaumont JL, Lemort N, Lorenzelli-Edouard L, Delplanque B, Beaumont V. Anti ethynilestradiol antibody activities in oral contraceptives users. Clin Exp antibody activities in oral

26 Beaumont JL, Lemort N, Beaumont V. Oral contraception, circulating human complexes, anti ethynilestradiol antibodies and thrombosis. Am J Reprod Immunol 1982;2:8-12.

27 Hardy-Godon S, Fredy D, Chodkiewitz JP, Perez J, Bories J. Angiography of cerebro-vascular accidents in patient taking contraceptive pills.-An analysis of 85 cases. $J$ Neuroradiol 1979;6:239-54.

28 Irey NS, Manion WC, Taylor HE. Vascular lesions in women taking oral contraceptives. Arch Pathol 1970;89: $1-8$

29 Grindall AB, Cohen RJ, Saul RF, Taylor JR. Cerebral infarction in young adults. Stroke 1978;9:39-42. 